\title{
Argon Cluster Ions Cleaning and Probing a Graphene Layer on Copper*
}

\author{
Kozo Mochiji ${ }^{\dagger}$ Norio Inui, Ryousuke Asa, and Kousuke Moritani \\ Graduate School of Engineering, University of Hyogo, \\ 2167, Shosha, Himeji-shi, Hyogo, 671-2280, Japan \\ (Received 2 March 2015; Accepted 31 March 2015; Published 18 April 2015)
}

\begin{abstract}
We demonstrate a method to clean and probe a graphene layer on copper by using cluster ions consisting of thousands of argon (Ar) atoms. Ar cluster ions colliding with a solid at kinetic energies below $\sim 5 \mathrm{eV} /$ atom dissociate into smaller cluster ions such as $\mathrm{Ar}_{2}^{+} \mathrm{or}_{\mathrm{Ar}_{3}^{+}}$, and the dissociation rate (ease of dissociation) depends on the physical property of the solid. We apply this phenomenon to probe a graphene layer on copper. Contaminants at the graphene surface are removed without damage to the surface by Ar cluster ion bombardment and the cleanliness of the surface is simultaneously probed by measuring the dissociation rate. This rate gradually approaches the value for a clean surface. After cleaning, the dissociation rate for pristine graphene on copper is five times lower than that for bare copper, indicating that the graphene layer acts as a buffer against the impact force of the cluster ion upon collision. Accordingly, the method can probe the quality of graphene, such as carbon coverage, by comparing the dissociation rate with that for bare copper. Furthermore, the method can probe the interface between graphene and copper, showing that the dissociation rate increases with increasing copper oxidation. The obtained experimental results are compared with simulated results obtained by molecular dynamics simulation for the collisions of an Ar cluster ion with graphene on copper. The combined results are discussed with respect to the utility of the proposed method for controlling the quality of graphene in the manufacture of electronic or mechanical devices. [DOI: 10.1380/ejssnt.2015.167]
\end{abstract}

Keywords: Argon cluster ion; Ion bombardment; Secondary ion mass spectroscopy; Computer simulations; Molecular dynamics; Graphene; Copper

\section{INTRODUCTION}

Graphene has attracted much attention because of its excellent electrical and mechanical properties [1-3]. The fabrication of electronic devices using graphene sheets requires contacts to metal electrodes or insulating substrates. The electronic properties of graphene on a metal or insulator are significantly different from the properties of freestanding graphene; consequently, it is important to have a good understanding of graphene-metal interfaces [4-6]. Furthermore, the cleaning of graphene surfaces is a key process in the fabrication of graphene-containing devices because surface contamination affects the properties of the device. However, methods using standard solvents such as acetone cannot remove all contaminants. Thermal desorption by high temperature annealing removes most contaminants but may thermally deform the graphene. Several techniques to overcome these shortcomings have been proposed $[7,8]$. Here, we propose a method to clean the surface of graphene on copper and to probe the cleanliness and coverage of the carbon using argon (Ar) cluster ions. This method can also probe the degree of oxidation of the copper surface without exfoliation of the graphene.

A neutral argon (Ar) cluster is held together by van der Waals forces and thus is fragile and easily dissociates into individual Ar atoms upon collision with a solid. In contrast, small Ar cluster ions such as $\mathrm{Ar}_{2}^{+}$or $\mathrm{Ar}_{3}^{+}$are much more stable because of the high binding energies between the Ar ion and the Ar atom(s). We have developed instrumentation for producing Ar cluster ions containing 100-10,000 Ar atoms and for mass analysis of the dissociation of the cluster ions upon impact with a solid

\footnotetext{
* This paper was presented at the 34th Annual Meeting of the Surface Science Society of Japan, Shimane Prefectural Convention Center (Kunibiki Messe), Matsue, Japan, November 6-8, 2014

$\dagger$ Corresponding author: mochiji@eng.u-hyogo.ac.jp
}

[9-12]. Importantly, this instrument lowers the average kinetic energy per Ar atom $\left(\mathrm{E}_{\mathrm{at}}\right)$ of the cluster ion to less than the binding energy of solids $(\lesssim 5 \mathrm{eV})$, which leads to nondestructive cleaning or probing. When $\mathrm{E}_{\mathrm{at}}$ of the incident cluster ion is adjusted to below $\sim 5 \mathrm{eV}$, the cluster ions dissociate into smaller cluster ions such as $\mathrm{Ar}_{2}^{+}$ and $\mathrm{Ar}_{3}^{+}$. The dissociation rate (ease of dissociation) of the cluster ions depends on the solid, or more exactly, the dissociation rate depends on physical properties such as the elastic coefficient of the solid [12]. We propose to employ this phenomenon to probe graphene on copper.

Here, we first demonstrate the simultaneous cleaning of a graphene surface and probing of its cleanliness by showing that the dissociation rate for graphene on a copper surface gradually approaches the value for a clean surface as surface contaminants are removed by Ar cluster ion bombardment. Second, we demonstrate the ability to probe the coverage of carbon atoms on the copper by comparing the dissociation rate for cleaned graphene on copper with that of bare copper. Third, we demonstrate the possibility of probing the interface between graphene and copper, showing that the dissociation rate increases as oxidation of the copper proceeds. Furthermore, in order to discuss the experimental results based on inter-atom interactions between Ar cluster ions and graphene on copper, we show the results of molecular dynamics simulation of collisions between Ar cluster ion, graphene and copper.

\section{EXPERIMENTAL}

\section{A. Methods}

Details of the experimental setup have been previously reported [9, 12]. Briefly, neutral Ar clusters were generated by supersonic expansion of Ar gas at a pressure of 0.5-1.2 $\mathrm{MPa}$ through a nozzle with a diameter of $0.1 \mathrm{~mm}$. The neutral clusters were ionized by electron bombard- 


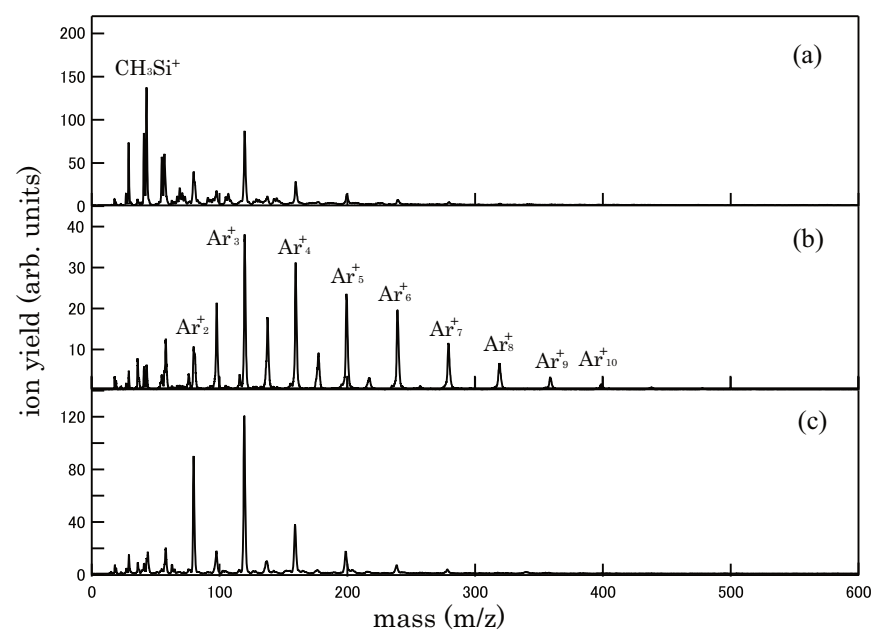

FIG. 1. Mass spectra of ions detected during bombardment with $\mathrm{Ar}_{1500}^{+}$at $3.3 \mathrm{eV} /$ atom of a graphene surface (a) before and (b) after pre-sputtering; (c) of a copper sample after presputtering. Both samples are kept in the vacuum-seal package for 1 day following production.

ment from a tungsten filament, and the resulting cluster ions were then extracted at an acceleration voltage of $2 \sim 10 \mathrm{kV}$. At this stage, the cluster size ranged from 1 (monatomic) to $\sim 7000$ atoms/cluster. The required cluster size was selected by employing a time-of-flight technique using two pairs of ion deflectors. The flight time of a cluster ion between the two pairs of ion deflectors depends on the cluster size. Therefore, clusters with a specific size can be extracted by adjusting the delay time between the two pairs of deflectors. In the present experiment, the peak cluster size $\mathrm{N}$ could be selected in the range 500 to 5000 atoms/cluster, and a cluster size resolution $(\mathrm{N} / \Delta \mathrm{N})$ of more than 10 was achieved by shortening the pulse width at the ion deflectors. As a result, $\mathrm{E}_{\mathrm{at}}$ could be varied in the range $0.5-20 \mathrm{eV}$ with an energy width of about $15 \%$. It should be emphasized that such controllability made it possible to detect differences in the dissociative behavior of cluster ions impacting different targets. The size-selected cluster ion beam (beam diameter: $\sim 0.5 \mathrm{~mm}$ ) was used to bombard the target at an incident angle of $45^{\circ}$ under a pressure of $3 \times 10^{-6} \mathrm{~Pa}$ at room temperature. Mass spectra of dissociated Ar cluster ions were measured using a reflectron-type mass analyzer with a flight tube length of $1.8 \mathrm{~m}$ (KNTOF-1800-SL203, Toyama, Kanagawa, Japan). Its mass resolution $(\mathrm{m} / \Delta \mathrm{m})$ was approximately 200 for $m / z=700$. The incident ionbeam current for each sample was measured using a picoammeter (6485, Keithley Instruments Inc. Cleveland, Ohio, USA).

The samples were single-layer graphene deposited on polycrystalline plates of copper with dimensions of $10 \times 10 \times 0.02 \mathrm{~mm}^{3}$ (hereafter called the graphene sample, Graphene Platform Co., Ltd., Tokyo, Japan). Graphene was deposited at the supplier by chemical vapor deposition (CVD) using a mixed gas of $\mathrm{H}_{2}, \mathrm{CH}_{4}$, and $\mathrm{Ar}$ at $\sim 1270 \mathrm{~K}$. Copper plates without a graphene deposit (hereafter called the copper sample) were used as a reference. The samples were inserted into a vacuum-seal pack- age containing an oxygen absorber soon after graphene deposition and were delivered to us. The samples were ultrasonically cleaned in methanol just before being placed in the ion bombardment chamber. The sample holder was made from stainless steel, and clasped the sample using a 1-mm-wide border around the front surface and covered the entire back surface of the sample. An Ar cluster ion containing several thousand atoms has a diameter of only a few nanometers [13]; thus, for all practical purposes, the sample size can be regarded as infinite. Accordingly, it is likely that elastic waves caused by ion impact freely propagate through the metal.

In order to analyze the chemical species at the sample surface, we measured the secondary ion mass spectra of the sample using Ar cluster ions with energies slightly higher than that used for the measurement of dissociated ions. The morphology of the sample surfaces was observed at the micrometer to nanometer scale, and at the atomic scale, using an atomic force microscope (AFM) (NanoScope3a-D3100, Veeco Ltd. Plainview, New York, USA) and a scanning tunneling microscope (STM) (USM1100, Unisoku Ltd. Osaka, Japan), respectively.

\section{B. Results and Discussion}

\section{Cleaning a graphene surface}

Figure 1(a) shows the mass spectra of ions detected from a graphene sample following collision with cluster ions containing $1500 \mathrm{Ar}$ atoms $\left(\mathrm{Ar}_{1500}^{+}\right)$at an acceleration voltage of $5 \mathrm{kV}\left(\mathrm{E}_{\mathrm{at}}=3.3 \mathrm{eV}\right)$. The vertical axis is the ion yield, which represents the detected ion count divided by the incident ion current. In addition to peaks due to dissociated Ar cluster ions, many additional peaks are observed in the spectrum, especially in the low-mass region $(\mathrm{m} / z \leq$ 100). Most are associated with contamination on the sample surface. Some of these peaks can be assigned to fragment ions $\left(\mathrm{CH}_{3} \mathrm{Si}^{+}\right)$of poly-dimethylsiloxane (PDMS). PDMS is often detected during surface analyses using techniques such as secondary ion mass spectrometry [14] and its likely sources are pump oil vapor in the vacuum chamber and resin in the sample case such as the vacuum seal package. Such contaminating species should be removed before measuring the dissociation of cluster ions because some collisional energy could be dissipated due to sputtering of these contaminants. Therefore, the samples were bombarded with Ar cluster ions with the same energies $\left(\mathrm{E}_{\mathrm{at}}=3.3 \mathrm{eV}\right)$ immediately prior to conducting measurements on dissociated ions. This is called the presputtering. Figures $1(\mathrm{~b})$ and 1(c) show the mass spectra for the graphene sample and copper sample, respectively, measured after the pre-sputtering with the ion dose of $1.5 \times 10^{12}$ ions $/ \mathrm{cm}^{2}$. In both spectra, the signals due to the contaminating species are remarkably reduced and a series of peaks associated with dissociated Ar cluster ions can be clearly observed. It should be noted that the relative strengths of these ion peaks are apparently different between the two samples; this will be discussed in more detail later. Furthermore, the peak due to monatomic argon ions $\left(\mathrm{Ar}^{+}\right)$is very small regardless of the prominence of $\mathrm{Ar}_{2}^{+}$or $\mathrm{Ar}_{3}^{+}$. The stability of these small Ar cluster ions comes from the covalent nature of the chemical bond 


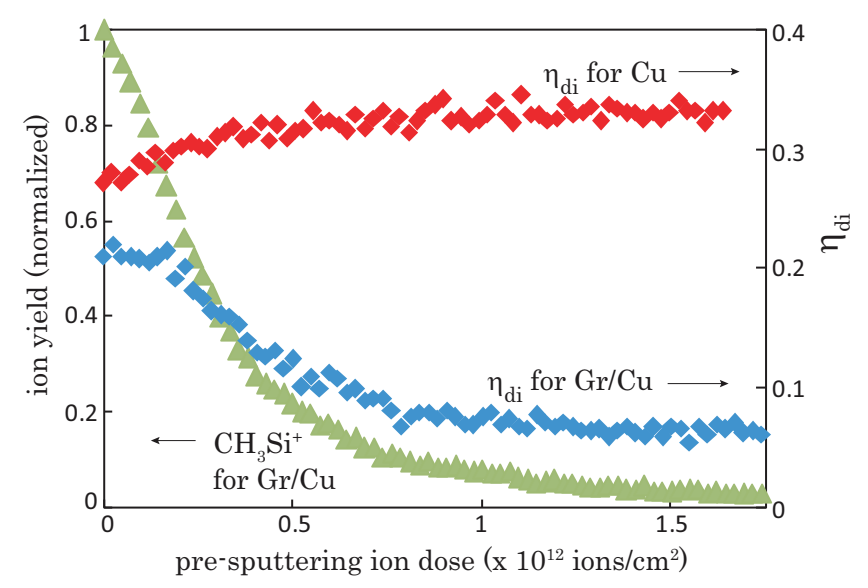

FIG. 2. Branching ratio $\left(\eta_{\mathrm{di}}\right)$ for graphene sample and copper sample, and ion yield of $\mathrm{CH}_{3} \mathrm{Si}^{+}$for graphene sample as a function of pre-sputtering ion dose. Both samples are kept in the vacuum-seal package for 1 day following production.

due to unfilled electron shells. Ikegami et al. reported that the charge of an Ar cluster ion is localized on the three central atoms, which form a trimeric ion core, and this core is much more stable than the shell of neutral Ar atoms surrounding it [15]. As a result, the energy corresponding to the dissociation of $\mathrm{Ar}_{3}^{+}$to $\mathrm{Ar}_{2}^{+}+\mathrm{Ar}$, and of $\mathrm{Ar}_{2}^{+}$to $\mathrm{Ar}^{+}+\mathrm{Ar}$, is 0.2 and $1.2 \mathrm{eV}$, respectively. Such a core-shell structure could also be present in large cluster ions consisting of thousands of $\mathrm{Ar}$ atoms. Here, we used the branching ratio for the dissociated ion yield, $\eta_{\mathrm{di}}$ $\left.=\left\{\left[\mathrm{Ar}^{+}\right]+\left[\mathrm{Ar}_{2}^{+}\right]\right\} / \sum \mathrm{Ar}_{n}^{+}\right](n \geq 1)$, obtained from the mass spectra, as an index representing the rate of dissociation of cluster ions.

$\eta_{\text {di }}$ and the ion yield of $\mathrm{CH}_{3} \mathrm{Si}^{+}$for the graphene sample and $\eta_{\text {di }}$ for the copper sample are plotted as a function of ion dose for the pre-sputtering in Fig. 2. The ion yield of $\mathrm{CH}_{3} \mathrm{Si}^{+}$is normalized at the beginning of the bombardment. $\eta_{\text {di }}$ and the ion yield of $\mathrm{CH}_{3} \mathrm{Si}^{+}$for the graphene sample decrease similarly with an increase in ion dose and $\eta_{\text {di }}$ approaches $\sim 0.07$. These results indicate that $\eta_{\text {di }}$ is sensitive to a very thin surface layer on the samples and pre-sputtering is effective for removing contaminating species and stabilizing $\eta_{\mathrm{di}}$. Consequently, pre-sputtering at a dose of $1.5 \times 10^{12}$ ions $/ \mathrm{cm}^{2}$ was routinely applied to all samples. AFM and STM were used to confirm that pre-sputtering at the above conditions has essentially no effect on the topography of the sample surface.

\section{Probing a clean graphene surface on copper}

Figure 2 also shows the $\eta_{\text {di }}$ curve for the copper sample. In contrast to the graphene sample, $\eta_{\text {di }}$ increases with an increase in ion dose and approaches 0.33 ; this is about five times higher than that for the graphene sample. As shown in Fig. 3, if the graphene sample was bombard at the higher energies $(\sim 10 \mathrm{eV})$ than $3.3 \mathrm{eV}, \eta_{\text {di }}$ rapidly decreased to the level for a clean surface $(\sim 0.07)$ but gradually increased again with an increase in ion dose and approached $\sim 0.33$, which exactly coincides with the value for the copper sample. The bombardment ion dose over

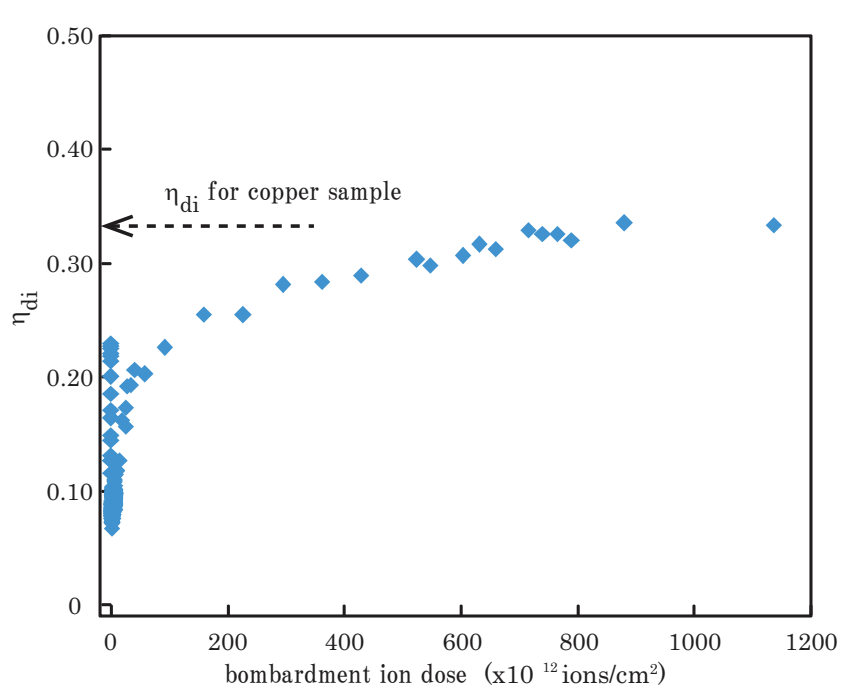

FIG. 3. Branching ratio $\left(\eta_{\mathrm{di}}\right)$ for graphene sample as a function of high-energy ion dose. The range of ion energies is $1 \sim 10$ $\mathrm{eV} /$ atom. The sample is kept in the vacuum-seal package for 1 day following production.

which $\eta_{\text {di }}$ increases shows that the graphene is being partially removed, exposing the underlying copper. Accordingly, it appears that $\eta_{\text {di }}$ probes the quality of graphene, such as carbon coverage or defect density.

We propose the following mechanism for the difference in the dissociation rate between the graphene sample and the copper sample. Upon collision between the cluster ion and the graphene sample, impinging Ar atoms in the cluster ion interact with tens of carbon atoms in the graphene surface and push them towards the copper substrate. This occurs since graphene is weekly bonded to copper; for example, the binding energy and equilibrium separation of the $\mathrm{C}-\mathrm{Cu}$ bond for $\mathrm{Cu}(111)$ is $0.03 \mathrm{eV}$ and $0.3 \mathrm{~nm}$, respectively [4]. As a result, part of the kinetic energy of the cluster ion could be dissipated through bending of the graphene sheet, resulting in graphene working as a buffer against the impact force of the cluster ion upon collision.

\section{Probing oxidation of the copper surface}

Our results showed that the value of $\eta_{\text {di }}$ significantly fluctuated depending on the graphene sample, presumably due to changes in the sample surface caused by aging. As mentioned before, the samples were placed in a vacuum-seal package soon after production at the supplier and were delivered sealed to us. We didn't measure the pressure in the package because the package was too small to install a vacuum gauge in it. Figure 4 shows the dependence of $\eta_{\text {di }}$ of several samples on the pre-sputtering ion dose. These samples differed in the time elapsed between production and measurement, and the gaseous environment prior to measurement. $\eta_{\text {di }}$ of most samples decreases with an increase in pre-sputtering ion dose but approaches to different levels for different samples. $\eta_{\text {di }}$ for samples kept in the vacuum-seal package until just before measurement is lower than that for a sample left in air for one day; however, $\eta_{\mathrm{di}}$ increases with an increase in 


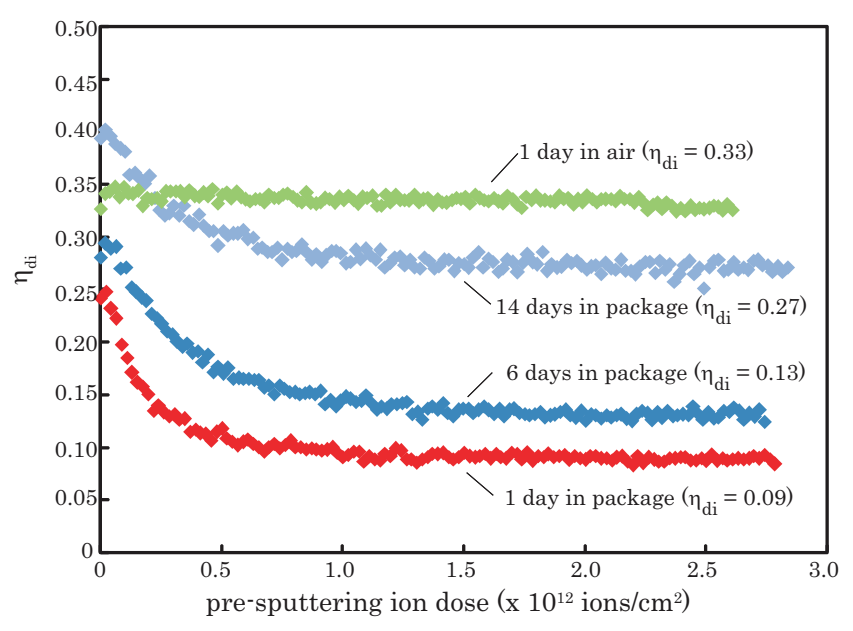

FIG. 4. Branching ratio $\left(\eta_{\mathrm{di}}\right)$ for graphene sample kept under various conditions as a function of pre-sputtering ion dose. The vacuum-seal package contains an oxygen absorber but the pressure in the package was not measured in this study.

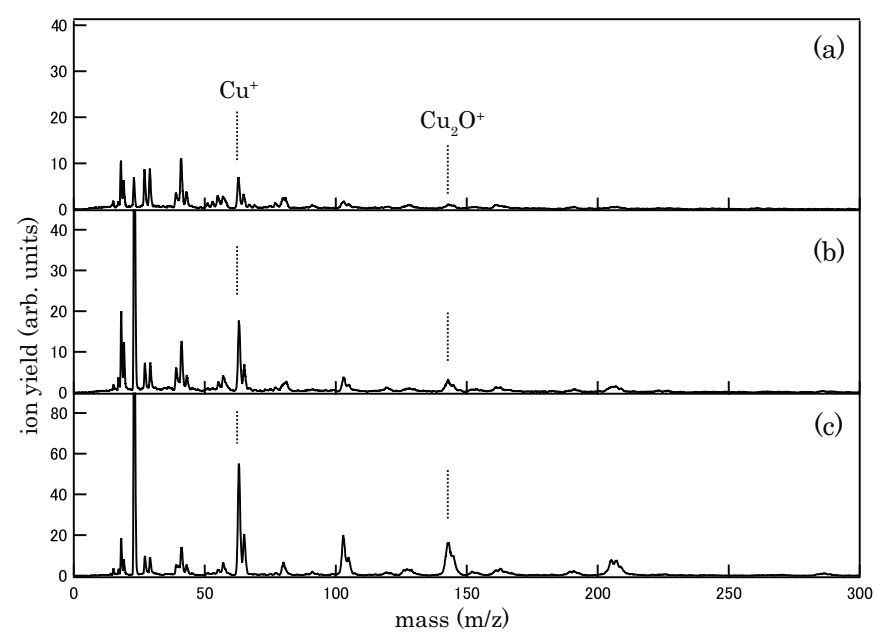

FIG. 5. Mass spectra of secondary ions from graphene samples, kept in a vacuum-seal package for (a) 1 day, (b) 14 days, and (c) left in air for 1 day following production. The ion energy of the Ar cluster ions for SIMS is $12.5 \mathrm{eV} /$ atom.

time in the sealed package. Secondary ion mass spectra (SIMS) of several samples measured at an $\mathrm{E}_{\mathrm{at}}$ of 12.5 $\mathrm{eV}$ are shown in Fig. 5. Copper ions and copper oxide ions were observed in all spectra and their ion yields were greatly increased by exposure to air. Figure 6(a) and 6(b) show AFM images of the samples kept in the vacuum-seal package for 4 days and left in air for one day, respectively. An alternating hill and valley structure is observed in both images and is probably due to faceting of the copper poly-crystal by high-temperature annealing $(\sim 1270 \mathrm{~K})$ during graphene deposition. The faceted surface becomes remarkably rough upon exposure to air. Taken together, these results indicate that the copper substrate becomes slightly oxidized after graphene deposition even in the vacuum-seal package and is much more rapidly oxidized in air. We didn't check the pressure in each sample package, but the pressure or the concentration of oxygen or water of the respective packages may
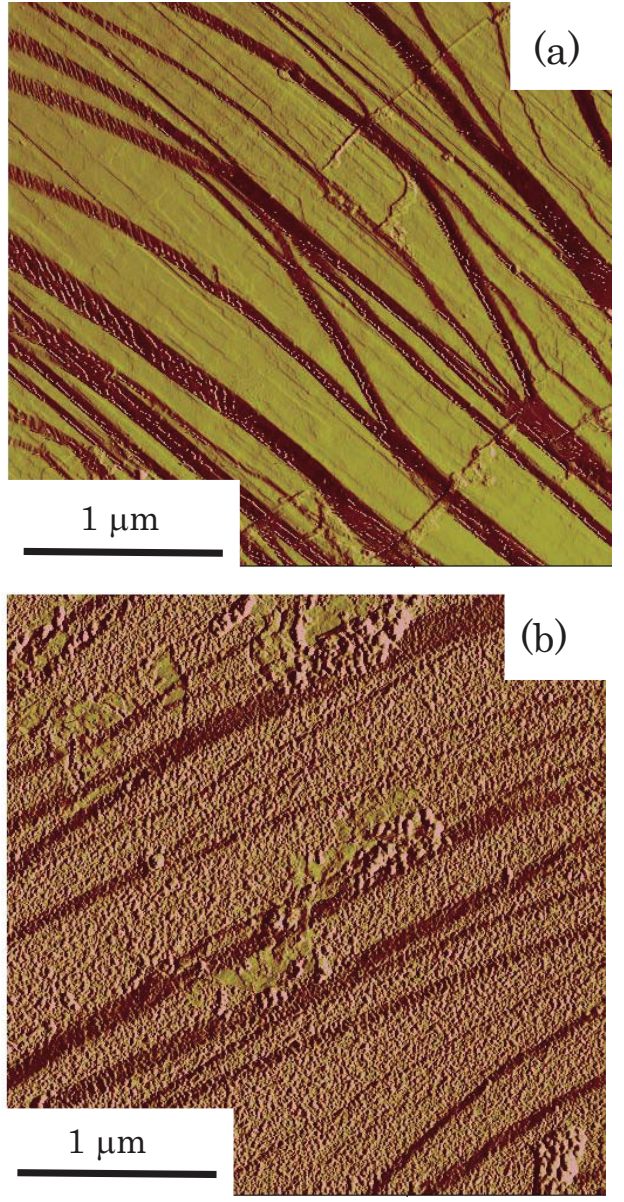

FIG. 6. AFM images of graphene samples, (a) kept in a vacuum-seal package for 4 days, and (b) left in air for 1 day following production. Both images are shown in amplitude data by tapping mode measurement. The average roughness (RMS) of the faceted surface were $1.9 \mathrm{~nm}$ and $2.7 \mathrm{~nm}$, for (a) and (b), respectively.

not change so much soon after the packaging at the supplier and thus oxidation increases with an increase in time in the package. The surface roughening seen in the AFM image could be due to the nucleation of copper-oxide islands, although this runs counter to the fact that pristine graphene is highly impermeable to any gas [16] and is robust against oxidation [17]. Recently, Schriver et al. reported that CVD graphene-covered copper is oxidized or corroded within a few months in ambient conditions [18] and proposed a mechanism in which $\mathrm{O}_{2}$ or $\mathrm{H}_{2} \mathrm{O}$ diffuses through graphene defects located mainly at the grain boundaries and reacts with copper to oxidize or corrode, assisted by the cathode effect of graphene. The oxidation observed in our samples could be similarly initiated at defects in the graphene layer.

Next, we considered why $\eta_{\text {di }}$ increases with an increase in copper oxidation. First, the binding energy of a $\mathrm{Cu}-$ $\mathrm{O}$ bond $(2.5 \sim 4.5 \mathrm{eV})$ at a copper oxide $\left(\mathrm{Cu}_{2} \mathrm{O}\right.$ or $\left.\mathrm{CuO}\right)$ surface is higher than that of a $\mathrm{Cu}-\mathrm{Cu}$ bond $(0.3 \sim 0.7 \mathrm{eV})$ at a bare copper surface [19]. This strengthens the interatomic potential at the surface of the substrate and promotes the dissociation of the cluster ions upon collision. We confirmed that $\eta_{\text {di }}$ for the copper sample left in air for 
one day was as high as $\sim 0.48$. Second, we believe that the average gap between the graphene layer and the substrate becomes narrower as oxidation progresses, making it difficult for graphene to deform and buffer the impact force of Ar cluster ion collision. Indeed, the dissociation behavior of Ar cluster ions sensitively probes the degree of oxidation, although further analyses of the structural changes await future investigation.

\section{SIMULATION OF AN AR CLUSTER ION IN COLLISION WITH GRAPHENE ON COPPER}

Molecular dynamics (MD) simulation was conducted to demonstrate that the dissociation rate of an Ar cluster ion depends on the depth of deformation of the solid surface. The dissociation of an Ar cluster by impact with a solid occurs within a time of $1 \mathrm{ps}$, making direct observation very difficult. Thus, MD simulation was used to investigate why the dissociation rate of an Ar cluster colliding with a graphene sheet is smaller than that for a collision with a bare copper substrate. Collisions of an Ar cluster containing 100 atoms were calculated using a classical MD simulation method, in which the total energy is conserved. We considered impacts with different configurations and obtained the dissociation rate by counting the atoms in a cluster ion after impact.

In this simulation system, an Ar cluster consists of an ionized $\mathrm{Ar}$ atom, $\mathrm{Ar}^{+}$, and 99 neutral Ar atoms. The interaction between the neutral Ar atoms is well-described by the Lennard-Jones potential

$$
\phi_{\alpha \beta}(r)=4 \epsilon_{\alpha \beta}\left[\left(\frac{\sigma_{\alpha \beta}}{r}\right)^{12}-\left(\frac{\sigma_{\alpha \beta}}{r}\right)^{6}\right],
$$

where $\epsilon_{\alpha \beta}$ and $\sigma_{\alpha \beta}$ are parameters of the potential between atomic species $\alpha$ and $\beta$. The parameters for argon $\epsilon_{\mathrm{ArAr}}$ and $\sigma_{\mathrm{ArAr}}$ are given in Table I. The interaction between an ionized Ar atom and a neutral Ar atom is more complicated, and no simple representation has been proposed. However, the most important parameters that characterize the interaction potential between $\mathrm{Ar}$ and $\mathrm{Ar}^{+}$ are the binding energy between $\mathrm{Ar}$ and $\mathrm{Ar}^{+}$and the equilibrium distance between the two atoms. These two properties can be obtained by choosing $\epsilon_{\alpha \beta}$ and $\sigma_{\alpha \beta}$ appropriately. According to a theoretical study of the potential energy of the trimer ion $\mathrm{Ar}_{3}^{+}$[15], the bond dissociation energy is $0.25 \mathrm{eV}$ and the bond length between atoms in a trimer ion is $0.260 \mathrm{~nm}$. Thus, to describe the interaction between $\mathrm{Ar}^{+}$and $\mathrm{Ar}$, we used the Lennard-Jones potential with $\epsilon_{\mathrm{ArAr}^{+}}=0.25 \mathrm{eV}$ and $\sigma_{\mathrm{ArAr}^{+}}=0.260 / 2^{1 / 6}$ nm. Here, we note that the Lennard-Jones potential has an energy minimum at $r=2^{1 / 6} \sigma_{\alpha \beta}$. We recognize that this expression is not a sufficient description for a trimer ion. The significant difference arises from the shape of the trimer ion. The stable configuration of the trimer ion calculated using the Lennard-Jones potential is an isosceles triangle, but the stable configuration conjectured by for a diatomic molecule is straight. This difference arises from the lack of interactions between bonds in our expression. Additionally, the interaction between $\mathrm{Ar}^{+}$and Ar depends on the number of neutral Ar atoms surrounding the $\mathrm{Ar}^{+}$ion; this is not taken into account using the
TABLE I. Parameters of Lennard-Jones potential.

\begin{tabular}{lccr}
\hline \hline$\alpha$ & $\beta$ & $\epsilon_{\alpha \beta}(\mathrm{eV})$ & $\sigma_{\alpha \beta}(\mathrm{nm})$ \\
\hline $\mathrm{Ar}$ & $\mathrm{Ar}$ & 0.01 & 0.341 \\
$\mathrm{Ar}$ & $\mathrm{Ar}^{+}$ & 0.25 & 0.231 \\
\hline \hline$\alpha$ & $\beta$ & $\epsilon_{\alpha \beta}(\mathrm{meV})$ & $\sigma_{\alpha \beta}(\mathrm{nm})$ \\
\hline $\mathrm{Ar}$ & $\mathrm{Cu}$ & 1.32 & 0.328 \\
$\mathrm{Ar}$ & $\mathrm{C}$ & 6.05 & 0.344 \\
$\mathrm{C}$ & $\mathrm{Cu}$ & 0.99 & 0.327 \\
\hline \hline
\end{tabular}

Lennard-Jones potential. In particular, the bonding energy of a dimer ion is $1.33 \mathrm{eV}$, and this strong bonding prevents the generation of a monomeric ion. Although few monomeric ions were observed in our experiment, a monomeric ion did appear in our MD simulation.

The interaction between copper atoms is described by the Morse potential:

$$
\phi_{\mathrm{M}}(r)=D_{\mathrm{M}}\left[e^{-2 \alpha\left(r-r_{0}\right)}-2 e^{-\alpha\left(r-r_{0}\right)}\right],
$$

where $D_{\mathrm{M}}=0.35 \mathrm{eV}, \alpha=0.134 \mathrm{~nm}^{-1}$, and $r_{0}=0.289 \mathrm{~nm}$ [20]. The interaction between carbon atoms is described by the Tersoff potential $[21,22]$. The Tersoff potential is a three-body potential and can describe the sputtering of a carbon atom. Although the Tersoff potential requires longer computation compared to other potentials (such as molecular mechanics), it allows verification of whether or not the present method provides a nondestructive cleaning and probing. The Tersoff potential parameters used in the MD simulations are identical to those in Table I of Ref. [22]. Other interactions are expressed using the LennardJones potential and the parameters are given in Table I [23]. A copper substrate with a face-centered cubic crystal structure has planar dimensions of $5.52 \mathrm{~nm} \times 5.52 \mathrm{~nm}$ and is $0.736 \mathrm{~nm}$ thick, and contains 2403 copper atoms. We employed two boundary conditions for the substrate. In the first boundary condition the atoms on the surface of the substrate are fixed except for the surface that faces the Ar cluster. In the second boundary condition, atoms on the surface of the substrate are fixed except for the surface that faces the Ar cluster and its opposite side. We refer to the first and second boundary conditions as the fixed boundary condition and the free boundary condition, respectively. A graphene sheet is a rectangle with dimensions $6.20 \mathrm{~nm}$ (zigzag edges) and $5.33 \mathrm{~nm}$ (armchair edges) and contains 1250 carbon atoms. The graphene sheet is located $0.326 \mathrm{~nm}$ above the copper surface. The carbon atoms on the periphery of the graphene sheet are fixed.

An Ar cluster ion is prepared using an annealing method. An ionized Ar is initially located at the center of a cluster containing 99 neutral Ar atoms, and the cluster is cooled from $30 \mathrm{~K}$ to $0.1 \mathrm{~K}$ in 0.1 ns intervals using the velocity scaling method. The Ar cluster collides with the surface of the substrate with an incident angle of $45^{\circ}$ and an incident energy of $3.3 \mathrm{eV}$ per atom. We used Gear's method with a time step of 0.1 fs. Figure 7 shows the dissociations of an Ar cluster colliding with a copper plate (left panel) and with a graphene sheet on copper (right panel) at $0,0.6$, and $1.2 \mathrm{ps}$. There is a large vertical deformation of the graphene sheet at $0.6 \mathrm{ps}$. The Ar atoms are more widely scattered in the collision with 

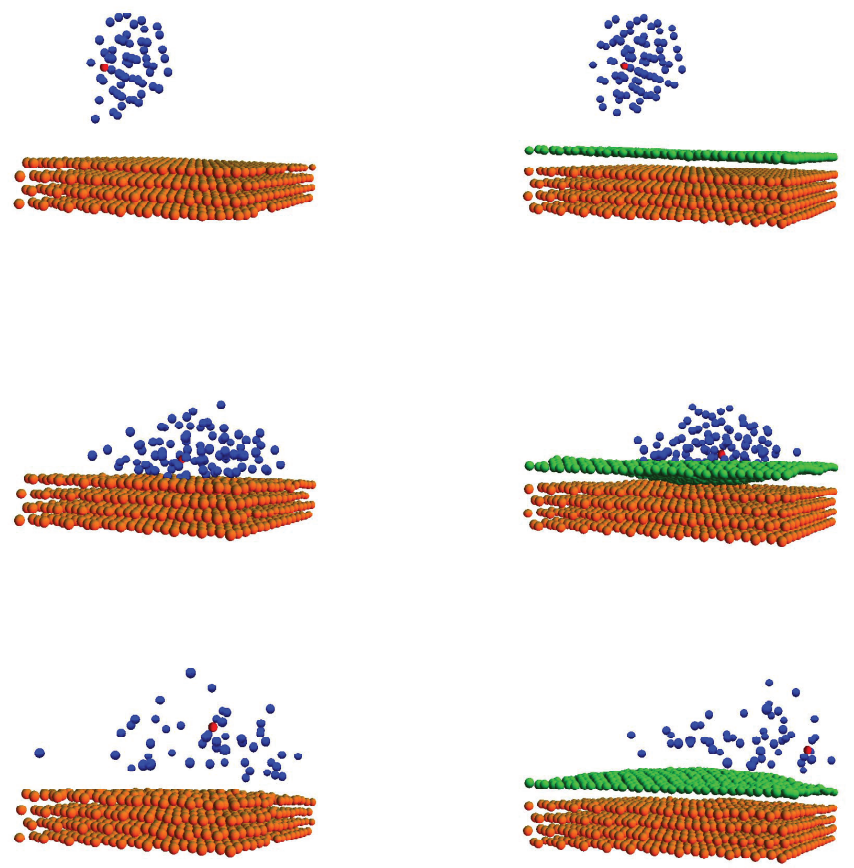

FIG. 7. Snapshots of a cluster consisting of 100 Ar atoms impacting a copper plate (left panel) and a graphene surface (right panel) at $0.6 \mathrm{ps}$ intervals from top to bottom. An Ar ion, a neutral Ar atom, a copper atom, and a carbon atom are represented by red, blue, orange, and green spheres, respectively.

the copper surface. We observed no sputtering phenomena from either the copper or the graphene surface in all trials.

To calculate the dissociation rate, we here introduce the energy of the cluster, $E_{c}^{n}$. We choose $n(n \geq 1)$ Ar atoms and label each atom from 1 to $n$. Let $r_{i}$ be the position of the $i$-th neutral Ar atom and $r_{i j}$ be the distance between the $i$-th atom and $j$-th atom. The energy of a cluster $E_{c}^{n}$ is defined by

$$
\begin{aligned}
E_{c}^{1}\left(r_{1}\right)=\phi_{\mathrm{ArAr}^{+}}\left(r_{01}\right) & +K_{1}, \\
E_{c}^{n>1}\left(r_{1}, r_{2}, \ldots, r_{n}\right) & =\sum_{i=1}^{n} \phi_{\mathrm{ArAr}^{+}}\left(r_{0 i}\right) \\
& +\sum_{i=1}^{n-1} \sum_{j=i+1}^{n} \phi_{\operatorname{ArAr}}\left(r_{i j}\right)+\sum_{i=1}^{n} K_{i},
\end{aligned}
$$

where $r_{0 i}$ is the distance between an Ar ion and the $i$-th atom, and $K_{i}$ is the kinetic energy of the $i$-th atom as measured by an observer at the Ar ion. We calculate $E_{\mathrm{c}}^{n}$ for all possible combinations by changing $n$. We determine the size of an Ar ion cluster by finding the maximum number, $n+1$, satisfying the conditions $E_{\mathrm{c}}^{n}<0$ and $E_{\mathrm{c}}^{n}<E_{\mathrm{c}}^{n-1}$.

The MD simulations were performed for 5 ps intervals over twenty trials in which the initial configuration of the cluster ion was randomly changed. We confirmed that, although the size of the cluster changes initially, the size of the Ar ion cluster does not change for times near 5 ps. The distance between Ar atoms for $t>5$ ps is much larger than $\sigma_{\alpha \beta}$ except inside the cluster ion itself, and very few

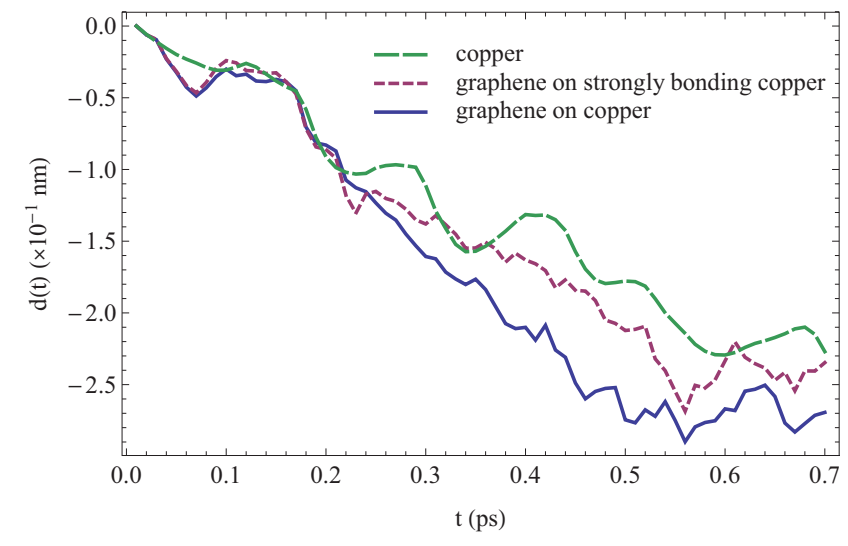

FIG. 8. Depth of deformation created by the impact of an Ar cluster against a copper substrate, a graphene sheet on a copper substrate and a graphene sheet on strongly bonding copper.

interactions occur between Ar atoms. Thus, if the size of an ion cluster is $n$ at $5 \mathrm{ps}$, we infer that $\mathrm{Ar}_{n}^{+}$is generated. The dissociation rate $\eta_{\mathrm{di}}=\sum_{n<3}\left[A r_{n}^{+}\right] / \sum_{n}\left[A r_{n}^{+}\right]$ where $\left[A r_{n}^{+}\right]$is the number of occurrences of $A r_{n}^{+}$, was obtained from 20 trials and was compared with the experimental results. The dissociation rates for the fixed boundary condition are 0.6 and 0.15 for the copper and graphene surfaces, respectively. For the free boundary condition, the dissociation rates are 0.4 and 0.1 for the copper and graphene surfaces, respectively. The dissociation rates for the copper surface are clearly larger than that for the graphene sheet under both boundary conditions and thus simulation result agrees with the experimental results. The difference in the dissociation rates for different boundary conditions is due to the finite size effects of the substrate. The dissociation rate for an infinitely thick substrate is probably between that for the fixed boundary condition and the free boundary condition.

We assume that a large deformation of the graphene sheet likely increases the concentration and suppresses the dissociation of an ion core. To examine this assumption, we consider a deformation of the surface. We define the depth, $d(t)$, of a deformation caused by an impact of an Ar cluster as

$$
\begin{aligned}
& d(t) \equiv-\left(\max \left\{z_{j}(t)\right\}-\min \left\{z_{k}(t)\right\}\right) \\
& \qquad \text { for } 1 \leq j, k \leq N_{s},
\end{aligned}
$$

where $z_{i}$ is the vertical position of the $i$-th atom in the surface at time $t$, and $N_{s}$ is the number of atoms in the surface. Here, we append a negative sign to express the fact that the surface initially moves downward. Figure 8 shows $d(t)$ obtained from simulations using the free boundary condition for a graphene sheet (blue solid line) and for the top surface of a copper substrate (green broken line). The same initial configuration of the Ar cluster was used for both targets. A larger deformation is created for a graphene sheet as compared to a copper surface. Impact with the copper substrate completely dissociated the $\mathrm{Ar}$ cluster, but a cluster ion consisting of four Ar atoms was created from the impact with the graphene sheet. 
If the deformation depth of the surface is the main factor that determines the dissociation rate, then an increase in the dissociation rate by the oxidization of a copper surface means a decrease in the deformation of the graphene sheet. Although the origin of the decrease in the deformation is unclear, we assume that the atoms at the surface of the substrate are strongly bonded by oxidization. To examine this assumption, we increased artificially the bonding energy between copper atoms by replacing the parameter $\mathrm{D}_{M}$ defined in equation (2), which determines the minimum of the interaction energy between copper atoms, with $5 \mathrm{D}_{M}(1.75 \mathrm{eV})$. We refer to this hypothetical substrate as strongly bonding copper. The red dotted line in Figure 8 shows the depth of the deformation of the graphene sheet on the strongly bonding copper using the free boundary condition. The Ar cluster ion completely dissociated upon impact. The absolute depth of the graphene sheet on the strongly bonding copper is small compared with that on ordinary copper. By increasing the bonding energy, the dissociation rate increased from 0.1 to 0.35 . This result suggests that the binding energy of atoms under a graphene sheet is reflected in the dissociation rate.

\section{CONCLUSIONS}

We demonstrate a method of cleaning and probing graphene on copper by using the branching ratio $\left(\eta_{\mathrm{di}}\right)$ of dissociated ions of Ar cluster ions. The cleanliness of the graphene surface is probed simultaneously with the removal of surface contaminants by cluster ion bombardment. The difference in the values of $\eta_{\mathrm{di}}$ after clean- ing between graphene-covered copper and bare copper probes the coverage of carbon on the copper. This method can also probe the degree of oxidized copper under the graphene. Molecular dynamics simulation assuming a Lennard-Jones potential for the ion core of the Ar cluster ion essentially reproduces the experimental results.

Contact with a metal electrode or insulating substrate is essential in electronic devices using a graphene sheet. The method proposed here can also be applied to the interface between graphene and metals other than copper or an insulator like silicon dioxide. The cleaning of graphene is also important for device fabrication and the proposed method could represent a promising technique to obtain clean, undamaged graphene surfaces. Application of the proposed method to mechanical devices such as sensors or protective films could allow simple, nondestructive probing of the mechanical strength of the graphene layer. Furthermore, the equipment needed for this method may be compatible with that for CVD fabrication of graphene in a vacuum. We believe this method could be used to control the quality of graphene in the manufacture of electronic or mechanical devices.

\section{ACKNOWLEDGMENTS}

This work was supported by a Grant-in-Aid for Scientific Research from the Japan Society for the Promotion of Science, and from the Development of System and Technology for Advanced Measurement and Analysis, Japan Science and Technology Agency. The authors would like to acknowledge T. Ikegami and H. Kuninaka for helpful discussions. The authors also thank N. Toyoda for his technical assistance for use of an AFM.
[1] K. S. Novoselov, A. K. Geim, S. V. Morozov, D. Jiang, Y. Zhang, S. V. Dubonos, I. V. Grigorieva, and A. A. Firsov, Science 306, 666 (2004).

[2] J. Yan, Y. Zhang, P. Kim, and A. Pinczuk, Phys. Rev. Lett. 98, 166802 (2007).

[3] A. R. Barton, J. Parpia, and G. H. Craighead, J. Vac. Sci. Technol. B 29, 050801 (2011).

[4] G. Giovannetti, P. A. Khomyakov, G. Brocks, V. M. Karpan, J. van den Brink, and P. J. Kelly, Phys. Rev. Lett. 101, 026803 (2008).

[5] M. Ishigami, H. J. Chen, G. W. Cullen, S. M. Fuhrer, and D. E. Williams, Nano Lett. 7, 1643 (2007).

[6] K. Nagashio, T. Yamashita, T. Nishimura, K. Kita, and A. Toriumi, J. Appl. Phys. 110, 024513 (2011).

[7] N. Lindvall, A. Kalabukhov, and A. Yurgens, J. Appl. Phys. 111, 064904 (2012)

[8] Z. Cheng, Q. Zhou, C. Wang, Q. Li, C. Wang, and Y. Fang, Nano Lett. 11, 767 (2011).

[9] K. Moritani, M. Hashinokuchi, J. Nakagawa, T. Kashiwagi, N. Toyoda, and K. Mochiji, Appl. Surf. Sci. 255, 948 (2008).

[10] K. Mochiji, M. Hashinokuchi, K. Moritani, and N. Toyoda, Rapid Commun. Mass Spectrom. 23, 648 (2009).

[11] N. Inui, K. Mochiji, and K. Moritani, Nanotechnology 19,
505501 (2008).

[12] K. Mochiji, N. Se, N. Inui, and K. Moritani, Rapid Commun. Mass Spectrom. 28, 2141 (2014).

[13] Y. Xiang, L. Cheng, W. Cai, and X. Shao, J. Phys. Chem. A 108, 9516 (2004).

[14] N. Tanaka, K. Moritani, T. Hirota, N. Toyoda, I. Yamada, N. Inui, and K. Mochiji, Rapid Commun. Mass Spectrom. 24, 1405 (2010).

[15] T. Ikegami and T. Kondow, J. Chem. Phys. 98, 3038 (1993).

[16] J. S. Bunch, S. S. Verbridge, and J. S. Alden, A. M. van der Zande, J. M. Parpia, H. G. Graighead, and P. L. McEuen, Nano Lett. 8, 2458 (2008).

[17] L. Liu, S. M. Ryu, M. R. Tomasik, and E. Stolyarova, N. Jung, M. S. Hybertsen, M. L. Steigerwald, L. E. Brus, and G. W. Flynn, Nano Lett. 8, 1965 (2008).

[18] M. Schriver, W. Regan, W. J. Gannet, A. M. Zaniewski, M. F. Crommie, and A. Zettl, ACS Nano 7, 5763 (2013).

[19] Y. R. Luo, Comprehensive Handbook of Chemical Bond Energies (CRC Press, Boca Raton, 2007).

[20] F. Milstein, J. Appl. Phys. 44, 3825 (1973).

[21] J. Tersoff, Phys. Rev. Lett. 56, 632 (1986).

[22] J. Tersoff, Phys. Rev. B 39, 5566 (1989).

[23] A. K. Rappe, C. J. Casewit, K. S. Colwell, W. A. Goddard III, and W. M. Skiff, J. Am. Chem. Soc. 114, 10024 (1992). 